\title{
Diseño de una ontología para el proceso de evaluación de las asignaturas técnico-científicas del Instituto Tecnológico de Orizaba
}

\author{
I. Colohua Cruz ${ }^{1}$, L. A. Reyes Hernández¹, G. Hernández Chan², \\ J. L Sánchez-Cervantes ${ }^{3}$ \\ ${ }^{1}$ División de Estudios de Posgrado e Investigación, \\ Instituto Tecnológico de Orizaba, \\ Orizaba, Veracruz, \\ México \\ ${ }^{2}$ Dirección de Ingeniería Eléctrica y Electrónica, \\ Instituto Tecnológico de Mérida, \\ Mérida, Yucatán, \\ México \\ ${ }^{3}$ Catedrático CONACYT comisionado al Instituto Tecnológico de Orizaba, \\ Orizaba, Veracruz, \\ México \\ icc90@outlook.com, 1.a.reyes.h@gmail.com, gandhi.hernandez@itmerida.mx, \\ jsanchezc@ito-depi.edu.mx
}

\begin{abstract}
Resumen. Este artículo presenta el diseño de una ontología para apoyar al proceso de evaluación de las asignaturas técnico-científicas (Cálculo Diferencial, Física, Química, entre otras) que se imparten a nivel licenciatura en el Instituto Tecnológico de Orizaba (ITO). El E-learning es una de las estrategias actuales que facilita el proceso de evaluación mediante el uso de entornos Web o plataformas digitales, los cuales comparten estrategias pedagógicas similares a las habituales aplicadas en cursos con modalidad presencial. Las preguntas que se aplican en la evaluación de asignaturas técnico-científicas por lo general tienen un resultado único, en su mayoría de forma numérica, y por lo tanto es conveniente generar respuestas que actúen como distractores al momento de aplicar un Quiz al alumno. La ontología que se presenta pretende especificar el dominio adecuado para la evaluación de las asignaturas técnico-científicas en el ITO. Para su desarrollo se siguió la metodología de ontologías Methontology y su codificación se realizó con Protégé.
\end{abstract}

Keywords: Methontology, Protégé, quiz, ontología, distractores asignaturas técnico-científicas. 


\title{
Design of an Ontology for the Evaluation Process in the Technical-Scientific Courses at Instituto Tecnológico de Orizaba
}

\begin{abstract}
This paper presents the design of an ontology to support the assessment process in the technical-scientific subjects (Differential Calculus, Physical, Chemical, among others) which are taught in the firsts years of bachelor's degree at Instituto Tecnológico de Orizaba (ITO). E-learning is one of the current strategies that facilitates the process of evaluation through the use of Web environments or digital platforms, which share pedagogical strategies similar to the usually applied in classroom courses. The questions that are applied in the evaluation of technical-scientific subjects usually have a single result, mostly of the time represented by a number, so it is convenient to generate answers that act as distractors when applying a quiz to a student. The ontology presented here pretends to specify the appropriate domain for the evaluation of the technicalscientific subjects at ITO. It was developed following the Methontology method and codified with Protégé.
\end{abstract}

Keywords: Methontology, Protégé, quiz, Ontology, distractors, technicalscientific courses.

\section{Introducción}

En la actualidad, los procesos de evaluación para las asignaturas técnico-científicas, por ejemplo Álgebra, Calculo, Física, Ecuaciones Diferenciales, Métodos Numéricos, entre otras que se imparten a nivel licenciatura en el Instituto Tecnológico de Orizaba (ITO) y en la mayoría de Instituciones de Educación Superior (IES), en donde se imparten programas de ingeniería, son laboriosos y extensos. Se ha observado que al aplicar los algoritmos Shuffle Option/Question (barajeo en preguntas y respuestas) en los exámenes de la materia de cálculo diferencial se cumple con los requisitos de las estrategias pedagógicas adecuadas para una evaluación, sin embargo, los entornos Web que aplican LMS (Learning Management System, Sistema de gestión de aprendizaje) como, por ejemplo, las plataformas Moodle y Chamilo, permiten la generación un Quiz pero no cumplen con las estrategias pedagógicas recomendadas [1]. No obstante, estas y otras plataformas Web enfocadas en la educación han tenido gran éxito puesto que cada día son más los usuarios que utilizan estas y otras herramientas en línea para promover y sacar provecho del E-learning (aprendizaje electrónico), el cual engloba a las aplicaciones y servicios que facilitan el proceso de enseñanza-aprendizaje, y que además se basan en el uso de las Tecnologías de la Información y la Comunicación (TIC) [2]. Además de implementar herramientas en línea para los procesos de evaluación, es necesario considerar técnicas para presentar un Quiz, el cual es una pequeña evaluación. Para ello, se recomienda que la evaluación contenga reactivos de tipo opción múltiple, que es una técnica que consiste en plantear una pregunta o problema, al cual se le denomina reactivo, que consta de un enunciado y una serie de respuestas, llamadas opciones. Entre estas respuestas hay una sola respuesta correcta, 
llamada solución, y otras incorrectas, conocidas como distractores. El número de respuestas opcionales en cada reactivo depende de la probabilidad que se acepte de que el alumno conteste bien al azar [3].

Los entornos Web enfocados a la gestión del conocimiento educativo desempeñan un papel importante para el E-learning, el cual surge de la necesidad de la educación y el aprovechamiento de la tecnología, en especial las TICs, para este fin. Además, el ELearning es uno de los pilares fundamentales para el auto aprendizaje y retroalimentación de algún tipo de conocimiento. Actualmente tiene un gran potencial en el mercado del software. En este sentido, es importante destacar que para el proceso de evaluación en línea no existen instrumentos que permitan medir los logros alcanzados por los estudiantes y se califiquen como "buenos o malos" ni tampoco hay ningún instrumento que cubra todas las necesidades de la evaluación, solo existen herramientas de apoyo para recopilar y administrar la información [4].

Por otra parte, el uso de las ontologías es común en áreas o dominios como los negocios, finanzas, Internet, medicina e industria y los dominios son usados para formalizar capitales intelectuales, integrar fuentes de datos, representar y organizar conocimiento [5]. También son empleados por el E-learning porque permiten visualizar de manera general los elementos necesarios en el dominio del mismo.

Por otra parte, para la elaboración de ontologías existen diversas metodologías, un ejemplo de ello es Methontology, la cual consta de 11 tareas que permiten llevar a cabo la elaboración de una ontología de forma correcta. Esta metodología permite construir ontologías en el nivel de conocimientos, y tiene sus raíces en las actividades como la gestión/administración, soporte y técnicas identificadas por el proceso de desarrollo de software propuesto por la organización IEEE [6].

Para fines prácticos es necesario que la ontología quede en un archivo con extensión .owl para ser implementada en algún sistema, y para algunos casos, es recomendable diseñar la ontología en una arquitectura modular, la cual se basa en la definición de una ontología de nivel superior que definirá las relaciones que deben aplicarse a un conjunto de sub-ontologías para cada sub-dominio [7].

Para la construcción de ontologías, una de las herramientas más conocidas es Protégé. Se trata de un software de código abierto que permite construir una ontología mediante su interfaz que ayuda al desarrollador en el proceso de construcción de la misma. Este software está disponible en su página oficial [8].

Los LMS están diseñados para apoyar al proceso de enseñanza-aprendizaje de manera virtual mediante un conjunto de herramientas que permiten la interacción y colaboración entre los actores del proceso (estudiantes, profesores, contenido), y tienen una orientación hacia el alumno, el profesor o para el proceso de aprendizaje. Los LMS que utilizan ontologías se dividen internamente en cuatro capas: interfaz (para el estudiante y/o profesor), servicios, contenidos (conocimientos o recursos) y actividades $[9,10]$.

El presente artículo presenta el diseño e implementación de una ontología para la administración y diseño de reactivos de evaluación de las asignaturas técnicocientíficas impartidas en el Instituto Tecnológico de Orizaba. Para la codificación de la ontología se utilizó Protégé y se siguió la metodología dictada por Methontology. 


\section{Metodología}

\subsection{Elementos de una ontología}

Los componentes de una ontología varían de acuerdo al dominio de interés y a las necesidades de dicho dominio. Por lo general, se encuentran los siguientes siete elementos [5].

1. Clases: Describen los conceptos del dominio y se organizan en taxonomías y por lo general se les aplican mecanismos de herencia.

2. Relaciones: Representan las interacciones entre los conceptos del dominio, por lo general contienen relaciones binarias. El primer elemento de la relación es el dominio y el segundo el rango.

3. Funciones: Son un tipo concreto de relación, donde se identifica un elemento mediante el cálculo a partir de otros elementos que se encuentren en la ontología.

4. Instancias: Representan objetos determinados de un concepto.

5. Taxonomía: Conjunto de conceptos organizados jerárquicamente.

6. Axiomas: Modelan sentencias que son siempre ciertas y permiten, junto con la herencia, inferir conocimiento que no esté indicado explícitamente en la taxonomía de conceptos.

7. Propiedades (slots). Son las características o atributos que describen a los conceptos.

\subsection{Tareas de methontology}

El diseño de la ontología que aquí se presenta está basado en las tareas definidas en la metodología Methontology, esto debido a que su completitud permite satisfacer los requerimientos propios del E-learning, por ejemplo motivar al alumno para procesar la información mientras se trabaja con los materiales de aprendizaje y que los medios de comunicación faciliten el procesamiento de la información para que el alumno obtenga el conocimiento requerido [11]. A continuación se describen las once tareas de conceptualización de la ontología.

\section{- Tarea 1: Glosario de términos}

Este glosario está constituido por los términos de interés del dominio E-learning determinados para el proceso de evaluación de las materias técnico-científicas del ITO. La Tabla 1 muestra los elementos (conceptos y atributos) más importantes. Cabe mencionar que hay términos que son entendidos como metadatos del estándar de Especificación de Interoperabilidad de Preguntas y Pruebas de IMS (IMS Question \& Test Interoperability Specification) que menciona el intercambio de temas, contenidos y resultados de datos entre herramientas de edición, bancos de reactivos, plataformas de aprendizaje y de evaluación [12]. No se detectaron sinónimos para los conceptos. 
Tabla 1. Extracto del glosario de términos.

\begin{tabular}{|c|c|c|}
\hline Nombre & Tipo & Descripción \\
\hline Image & Atributo & $\begin{array}{l}\text { Es un elemento que representa una imagen o una gráfica } \\
\text { para el Item. }\end{array}$ \\
\hline Text & Atributo & Es el enunciado que forma parte del Item. \\
\hline Distractor & Atributo & $\begin{array}{l}\text { Son aquellas respuestas que presentan errores de } \\
\text { pensamiento que ocurren con frecuencia y que parecen } \\
\text { verdaderas. }\end{array}$ \\
\hline Correct Answer & Atributo & Es la respuesta correcta a un Item. \\
\hline Result & Atributo & Es el puntaje obtenido del Quiz. \\
\hline Item & Concepto & Es una pregunta estructurada que forma parte de un Quiz \\
\hline Subject & Concepto & Es la asignatura que imparte un maestro. \\
\hline Topic & Concepto & $\begin{array}{l}\text { Los temas que están diseñados con base en los contenidos } \\
\text { de las asignaturas. }\end{array}$ \\
\hline Student & Concepto & Persona que presenta un Quiz. \\
\hline Quiz & Concepto & Es el conjunto de Item's para ser evaluados. \\
\hline Concentrated & Concepto & $\begin{array}{l}\text { Es el conjunto de resultados de varios Quiz que presenta el } \\
\text { Student. }\end{array}$ \\
\hline Group & Concepto & $\begin{array}{l}\text { Es el conjunto de Student's de una asignatura que presentan } \\
\text { un Quiz. }\end{array}$ \\
\hline Teacher & Concepto & $\begin{array}{l}\text { Es la persona responsable de impartir una materia (Subject) } \\
\text { y elaborar un Quiz. }\end{array}$ \\
\hline
\end{tabular}

\section{- Tarea 2: Taxonomía de conceptos}

La Fig. 1 muestra la jerarquía de los conceptos que fue definida a partir del glosario de términos de acuerdo al dominio de E-learning.

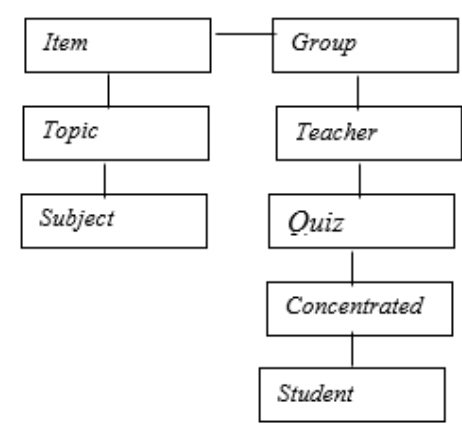

Fig. 1. Taxonomía de conceptos.

\section{- Tarea 3: Diagrama de relaciones binarias}

La Fig. 2 muestra el diagrama que establece los tipos de relaciones entre los conceptos antes definidos. Se omiten los atributos de los mismos. 


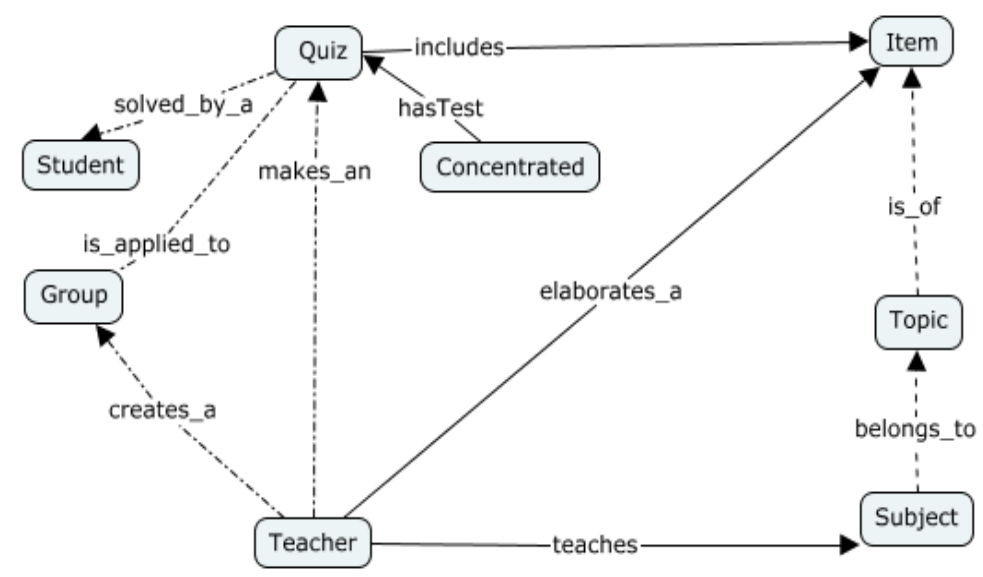

Fig. 2. Diagrama de relaciones binarias.

\section{- Tarea 4: Construir el diccionario de conceptos}

En la Tabla 2 se muestran los conceptos que se determinaron como los más relevantes en el proceso de E-learning. Estos conceptos se consideran los de mayor importancia debido a que, en la dinámica correspondiente al proceso de elaboración de evaluaciones, los actores principales son los profesores, ya que son ellos quienes definen y agregan reactivos a los exámenes, sin embargo, esto conlleva otras actividades anteriores, por ejemplo, crear los grupos y crear los exámenes con base en los contenidos de las asignaturas que hayan impartido. Por otra parte, el Quiz es por sí mismo, un elemento fundamental, ya que se trata del medio o la herramienta mediante la cual se logra la evaluación.

Tabla 2. Diccionario de conceptos.

\begin{tabular}{|c|c|c|}
\hline Concepto & & elaciones \\
\hline Teacher & $\begin{array}{l}\text { creates_a -> Group } \\
\text { makes_an-> Quiz }\end{array}$ & $\begin{array}{c}\text { elaborates_a->Item } \\
\text { teaches->Subject }\end{array}$ \\
\hline Quiz & $\begin{array}{l}\text { is_applied_to-> Group } \\
\text { includes->Item }\end{array}$ & solved_by_a->Student \\
\hline
\end{tabular}

\section{- Tarea 5: Detallar relaciones binarias}

Para cada relación se especifica el nombre, conceptos fuente (A) y destino (B), cardinalidad del dominio de E-learning. Véase Tabla 3.

Tabla 3. Detalles de las relaciones binarias.

\begin{tabular}{|l|l|l|l|}
\hline Nombre & Concepto A & Concepto B & Cardinalidad \\
\hline teaches & Teacher & Subject & $1: 1 \ldots{ }^{*}$ \\
\hline creates_a & Teacher & Group & $1: 1 \ldots *$ \\
\hline
\end{tabular}


Diseño de una ontología para el proceso de evaluación de las asignaturas técnico-científicas ...

\begin{tabular}{|l|l|l|l|}
\hline makes_an & Teacher & Quiz & $1: 1 \ldots *^{*}$ \\
\hline elaborates_a & Teacher & Item & $1: 1 \ldots *^{*}$ \\
\hline includes & Quiz & Item & $1: *$ \\
\hline is_applied_to & Quiz & Group & $1: 1 \ldots{ }^{*}$ \\
\hline solved_by_a & Quiz & Student & $1 \ldots *^{*} 1 \ldots *^{*}$ \\
\hline is_of & Item & Topic & $1: 1$ \\
\hline belongs_to & Topic & Subject & $1 \ldots *_{1} 1$ \\
\hline hasTest & Concentrated & Quiz & $1 \ldots *^{*} 1$ \\
\hline
\end{tabular}

\section{- Tarea 6: Definir los atributos de instancias}

Aquí se definen algunos atributos de instancias. Cabe mencionar que todas las clases tienen por lo menos una instancia, a continuación, en la Tabla 4 se muestran los más relevantes. NombreStudent representa un atributo de Student, el cual es el nombre completo del alumno, IdEstudent representa la matrícula del estudiante, los demás atributos que pertenecen a Item esta descritos en la Tabla 1.

Tabla 4. Atributos de instancia de las clases Student e Item.

\begin{tabular}{|l|l|l|l|l|}
\hline Atributo & Concepto & Tipo de Valor & Rango & Cardinalidad \\
\hline NombreStudent & Student & String & ilimitado & 1 \\
\hline IdEstudent & Student & String & ilimitado & 1 \\
\hline Correct Answer & Item & int & números reales & 1 \\
\hline Distractor & Item & int & números reales & 3 \\
\hline Image & Item & & ilimitado & $0: 1$ \\
\hline Text & Item & String & ilimitado & 1 \\
\hline
\end{tabular}

\section{- Tarea 7: Detallar atributos de clase}

La Tabla 5 muestra los atributos de clase, los cuales representan características generales de un concepto, es decir, todas las instancias del concepto tendrán el mismo valor para ese atributo.

Tabla 5. Atributos de clase Quiz.

\begin{tabular}{|l|l|l|l|}
\hline Atributo & Concepto & Tipo de Valor & Rango de Valores \\
\hline Mínimo de Item's por Quiz & Quiz & Item & 5 \\
\hline
\end{tabular}

\section{- Tarea 8: Detallar constantes}

Para cada constante se especifica el nombre, tipo de valor, valor y unidad de medida. Esta tarea no aplica para el dominio del E-learning.

\section{- Tarea 9: Detallar axiomas formales}

Los axiomas describen restricciones del modelo, así como las características que debe cumplir algún concepto. En la Tabla 6 se muestra el nombre, descripción, expresión lógica, conceptos o relaciones, y variables de los axiomas que intervienen. 
Tabla 6. Axiomas formales.

\begin{tabular}{|c|c|c|c|c|}
\hline Nombre & Descripción & Expresión Lógica & $\begin{array}{l}\text { Conceptos/ } \\
\text { Relaciones }\end{array}$ & $\begin{array}{l}\text { Varia- } \\
\text { bles }\end{array}$ \\
\hline NoAnswer & $\begin{array}{l}\text { Un distractor es una } \\
\text { respuesta que no es } \\
\text { correcta }\end{array}$ & $\begin{array}{c}\text { NO [ EXISTE( } \\
\text { Item }(? \mathrm{x}) \mathrm{Y} \\
\text { ?x.Correct } \\
\text { Answer=false; ) ] }\end{array}$ & $\begin{array}{l}\text { Item } \\
\text { Correct Answer }\end{array}$ & $? \mathrm{x}$ \\
\hline Anwser & $\begin{array}{l}\text { De una pregunta } \\
\text { solo hay una } \\
\text { respuesta correcta }\end{array}$ & $\begin{array}{l}\text { EXISTE( } \\
\text { Item }(\text { ?x) Y } \\
\text { ?x.Correct Aswer } \\
\text { =true ; ) }\end{array}$ & $\begin{array}{l}\text { Item } \\
\text { Correct Answer }\end{array}$ & $? \mathrm{x}$ \\
\hline Question & $\begin{array}{l}\text { De un tema puede } \\
\text { haber varias } \\
\text { preguntas }\end{array}$ & N/A & $\begin{array}{l}\text { Topic } \\
\text { Item }\end{array}$ & N/A \\
\hline Assessment & $\begin{array}{l}\text { Una evaluación se } \\
\text { compone de una } \\
\text { serie de preguntas }\end{array}$ & N/A & $\begin{array}{l}\text { Quiz } \\
\text { Item } \\
\text { Distractor }\end{array}$ & N/A \\
\hline
\end{tabular}

\section{- Tarea 10: Definir reglas}

En esta tarea se definen las reglas necesarias para la ontología de E-learning, se definen en la Tabla 7, recordando que para cada regla, se especifica el nombre, descripción, expresión y conceptos.

Tabla 7. Reglas.

\begin{tabular}{|c|c|c|c|}
\hline Nombre & Descripción & Expresión & Conceptos \\
\hline $\begin{array}{l}\text { Barajear } \\
\text { opciones }\end{array}$ & $\begin{array}{l}\text { Se barajean las opciones } \\
\text { siempre y cuando el } \\
\text { atributo no se haya } \\
\text { seleccionado. }\end{array}$ & $\begin{array}{l}\text { Si [Quiz (?x) Y } \\
\text { Item (?y) Y ?x include ?y } \\
\text { ] ENTONCES [ } \\
\text { chose(?x, ?y) } \\
\text { shuffe (?x, ?y)] }\end{array}$ & $\begin{array}{l}\text { Quiz } \\
\text { Item }\end{array}$ \\
\hline $\begin{array}{l}\text { Mostrar } \\
\text { Item's } \\
\text { más } \\
\text { frecuentes. }\end{array}$ & $\begin{array}{lll}\text { Muestra } & \text { los } & \text { Item's } \\
\text { utilizados } & \text { con } & \text { mayor } \\
\text { frecuencia. } & & \\
\end{array}$ & $\begin{array}{l}\text { Si }[\text { Quiz }(? \mathrm{x}) \mathrm{Y} \\
\quad \text { Item }(? \mathrm{y}) \mathrm{Y} \\
\text { chose }(? \mathrm{x}, ? \mathrm{y})] \\
\text { ENTONCES } \\
\text { ?y.masUsu=?y. masUsu + } 1\end{array}$ & $\begin{array}{l}\text { Quiz } \\
\text { Item }\end{array}$ \\
\hline $\begin{array}{l}\text { Mostrar } \\
\text { Item's } \\
\text { menos } \\
\text { frecuentes. }\end{array}$ & $\begin{array}{lll}\text { Muestra } & \text { los } & \text { Item's } \\
\text { utilizados } & \text { con } & \text { menor } \\
\text { frecuencia. } & & \\
\end{array}$ & $\begin{array}{l}\text { Si }[\text { Quiz }(? \mathrm{x}) \mathrm{Y} \\
\quad \text { Item }(? \mathrm{y}) \mathrm{Y} \\
\text { chose }(? \mathrm{x}, ? \mathrm{y})] \\
\text { ENTONCES } \\
\text { ?y.menUsu=?y.menUsu + } 1\end{array}$ & $\begin{array}{l}\text { Quiz } \\
\text { Item }\end{array}$ \\
\hline
\end{tabular}

\section{- Tarea 11: Definir instancias}

En esta tarea se definen las instancias de conceptos de la ontología, cada instancia cuenta con nombre, nombre del concepto al que pertenece y los valores de sus atributos de instancia, si se conocen. Esta tarea no aplica por el momento hasta la creación de la ontología del dominio de E-learning. 


\section{Resultados y discusión}

El proceso de evaluación en línea es una estrategia que permite saber el grado de aprendizaje de un alumno en un tema específico. Un buen Quiz cuenta con distractores que cumplen con la parte pedagógica de un modelo educativo, para ello se analizaron diversas formas de generar un distractor. Específicamente en el área de las matemáticas, por ejemplo en álgebra, se ha observado que uno de los errores más comunes que cometen los estudiantes es no respetar el orden de los operadores o de los paréntesis cuando tratan de resolver alguna ecuación, por lo que una forma de crear distractores es cambiando el orden de los operadores o de los paréntesis.

Siguiendo la metodología propuesta por Methontology, lo que se obtuvo al final de las 11 tareas descritas anteriormente fue la identificación de los conceptos principales de E-learning y la relación entre ellos, los cuales fueron tomados como base para la obtención de un diseño conceptual de una ontología que sirve de soporte para el proceso de creación de evaluaciones de asignaturas técnico-científicas. Finalmente, con base en el modelo obtenido, se procedió a la codificación de la ontología utilizando el software Protégé.

Tabla 8. Codificación en Protégé.

\begin{tabular}{|l|l|l|}
\hline \multicolumn{2}{|c|}{ Concepto } & \multicolumn{2}{c|}{ Relaciones } \\
\hline Teacher & $\begin{array}{l}\text { creates_a }->\text { Group } \\
\text { makes_an-> Quiz }\end{array}$ & $\begin{array}{c}\text { elaborates_a->Item } \\
\text { teaches->Subject }\end{array}$ \\
\hline Quiz & $\begin{array}{l}\text { is_applied_to-> Group } \\
\text { includes->Item }\end{array}$ & solved_by_a->Student \\
\hline
\end{tabular}

El siguiente paso fue la codificación de la ontología con la herramienta Protégé, porque permite generar código de un lenguaje ontológico. A continuación se muestra la descripción de las pantallas principales donde se visualiza la creación de la misma. La Fig. 3 muestra el panel de clases, la cual, permite definirlas, establecer la jerarquía y restricciones. En la Fig. 4 se visualiza la creación de las relaciones binarias.

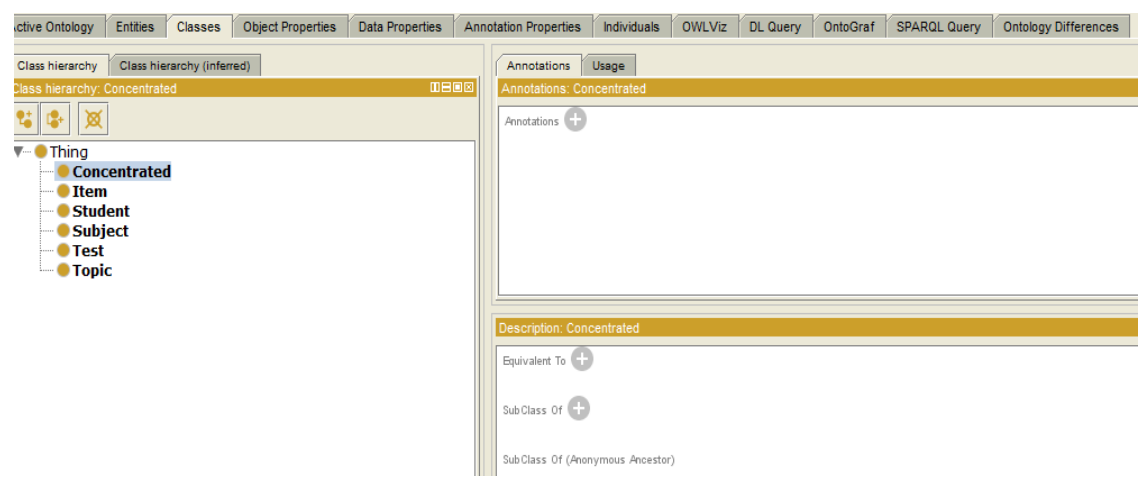

Fig. 3. Creación de clases del dominio de E-learning en Protégé. 


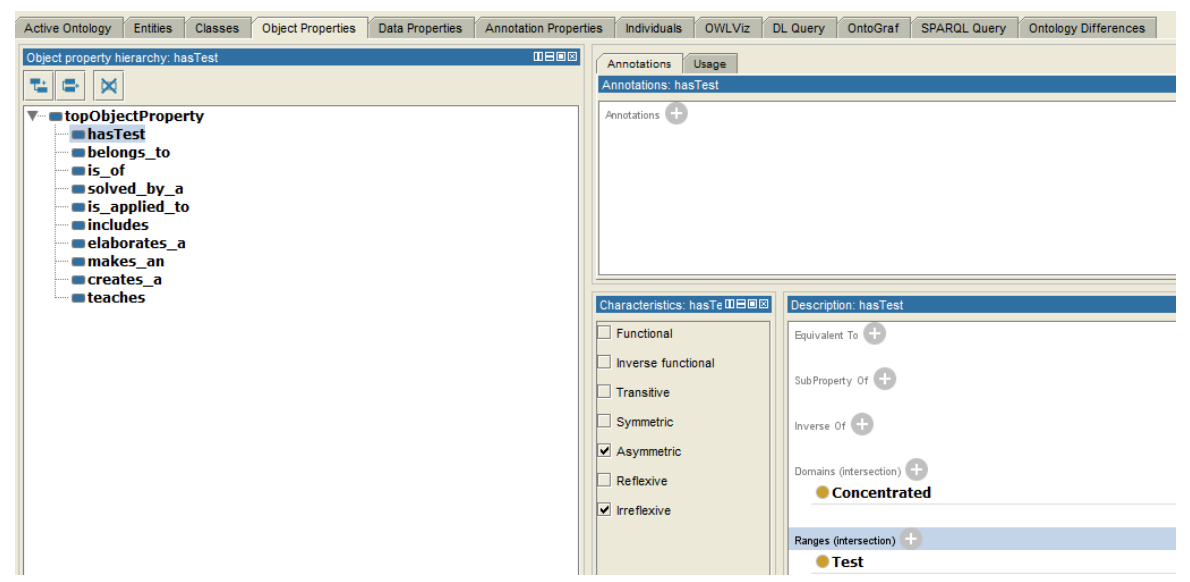

Fig. 4. Relaciones binarias del dominio E-learning.

Para la comprobación del diseño de la ontología se agregaron algunos individuos (instancias) para comprobar que las relaciones y las clases estén correctamente diseñadas. En la Fig. 5 se observan algunos los individuos de los conceptos de Teacher, Topic, Student y Subject con sus respectivos atributos de clase que se les proporcionaron para realizar algunas consultas basadas en SPARQL.

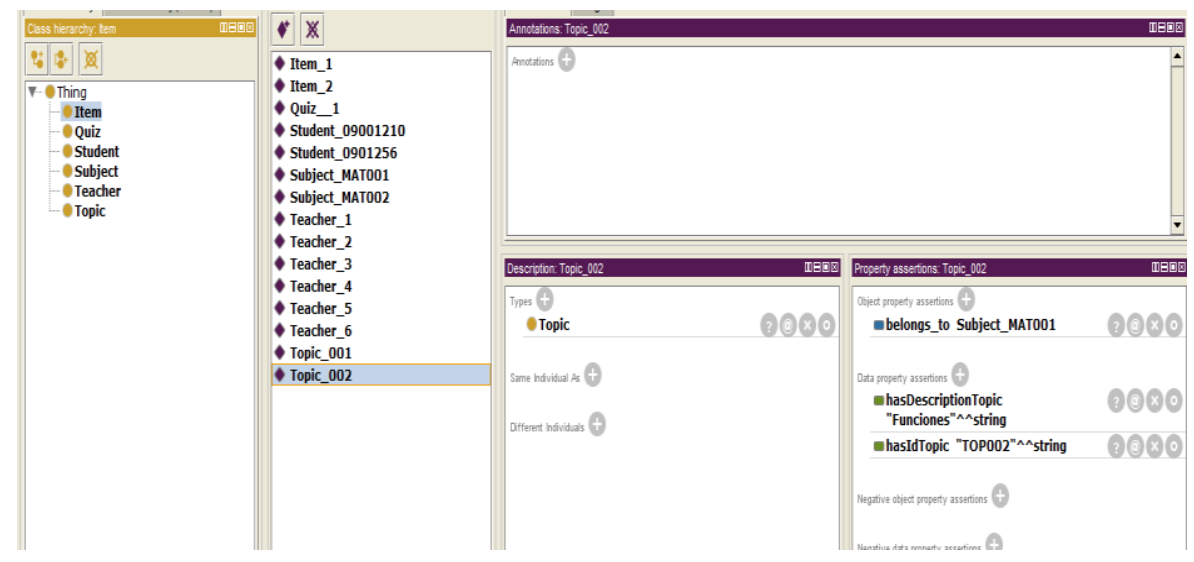

Fig. 5. Instancias agregadas a la ontología del dominio E-learning.

El lenguaje SPARQL (Protocol and RDF Query Language) está diseñado para elaborar consultas sobre datos representados para RDF (Resource Description Framework), el cual contiene funciones de consultas requeridas y opcionales para ser utilizadas al momento de expresar los resultados a través de diversas fuentes de da-tos [13]. A continuación, en la Fig. 6, Fig. 7 y Fig. 8 se muestran los resultados de algunas consultas que se elaboraron sobre la ontología y su respectivo código escrito en SPARQL. Es Importante mencionar que hemos omitido los prefijos en las consultas basadas en SPARQL para optimizar espacio en el presente artículo. 
Diseño de una ontología para el proceso de evaluación de las asignaturas técnico-científicas ...

a) Buscar el id y nombre de los profesores.

\section{SELECT ?id ?name}

WHERE \{ ?x rdf:type itoTest:Teacher. ?x itoTest:hasIdTeacher ?id.

?x itoTest:hasNameTeacher ?name

\}

b) Buscar el id y descripción de las asignaturas técnico-científicas.

\section{SELECT ?id ?description}

WHERE $\{$ ?x rdf:type itoTest:Subject.

?x itoTest:hasIdSubject?id.

?x itoTest:hasDescriptionSubject ?description

$$
\text { \} }
$$

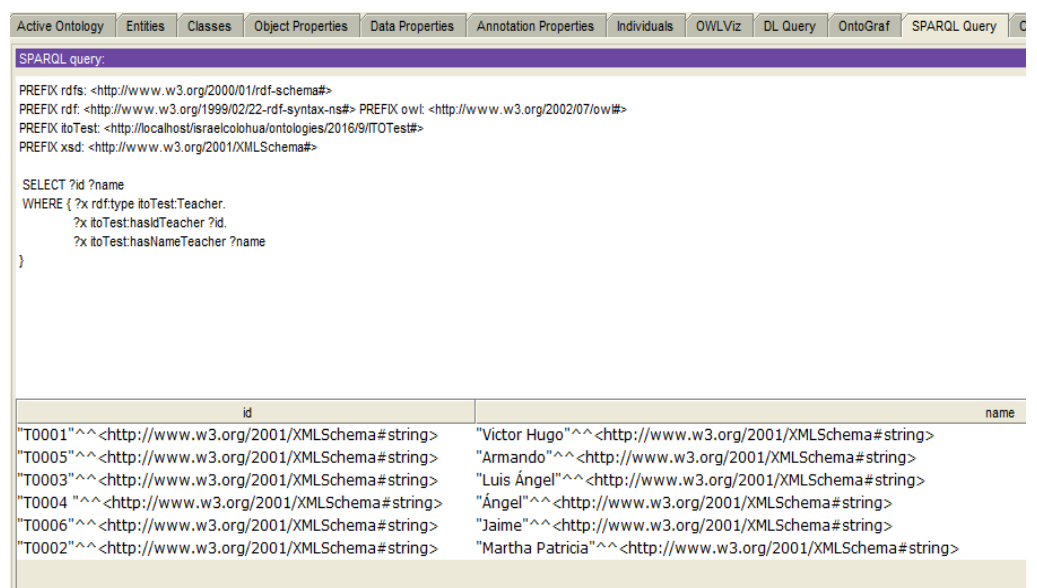

Fig. 6. Resultado de la consulta sobre los profesores.

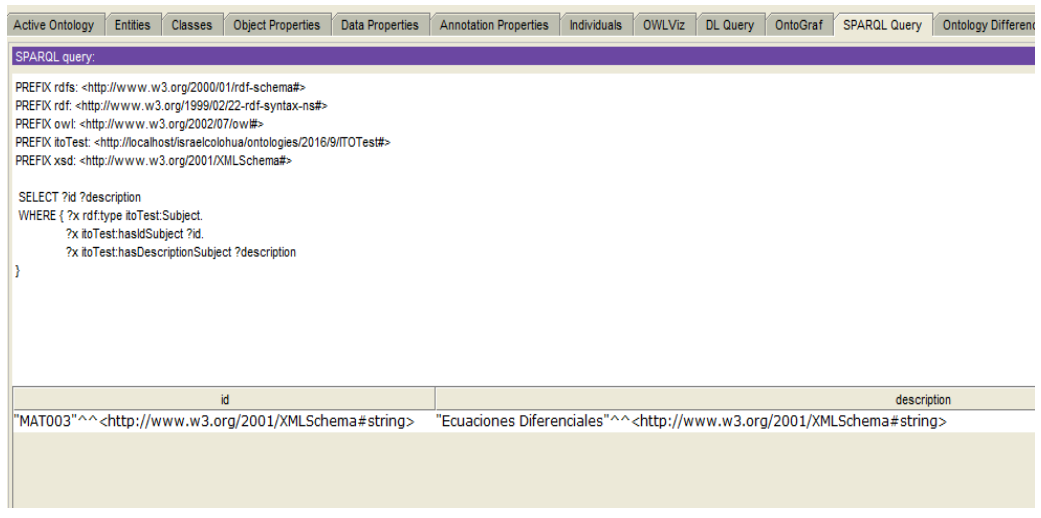

Fig. 7. Resultado de la consulta en las asignaturas. 
c) Buscar la descripción y respuesta correcta de los item’s disponibles

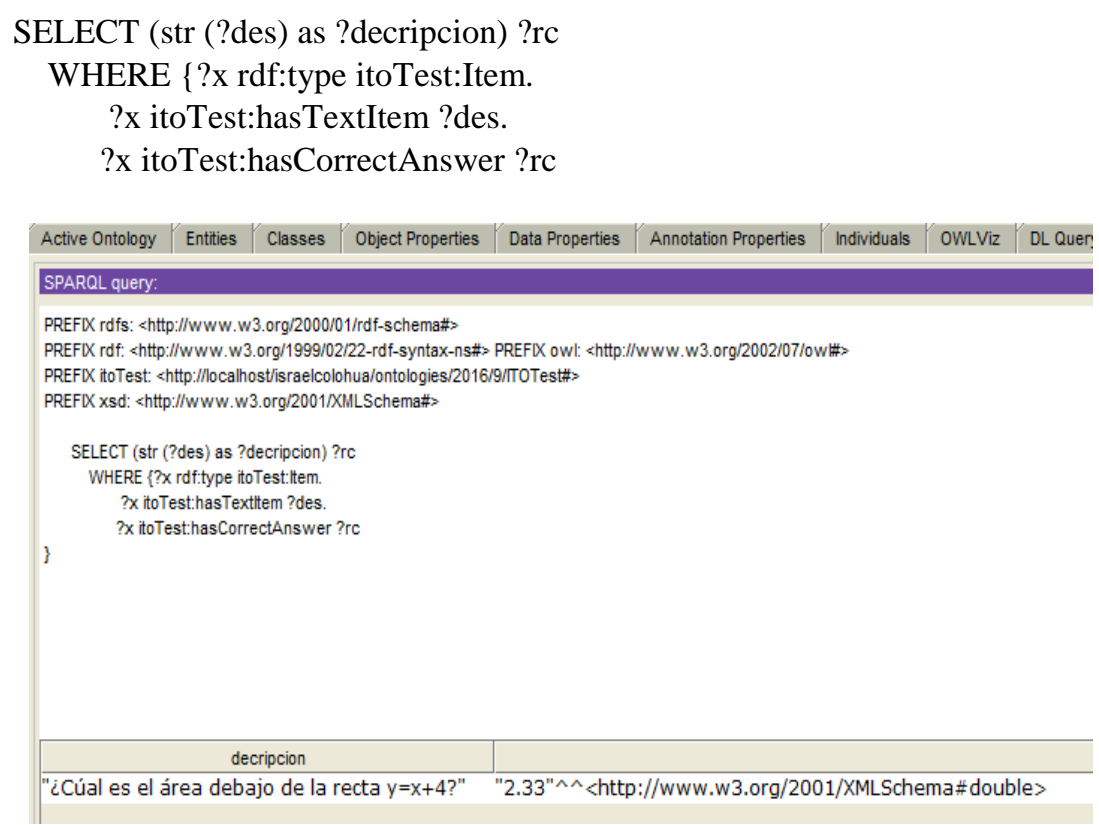

Fig. 8. Resultado de la consulta en los Item's.

\section{Conclusiones}

En la actualidad, los contenidos, materiales y cursos que son impartidos en línea son ofrecidos al estudiante a través de plataformas de E-learning, que comúnmente son aplicaciones Web que aplican herramientas y servicios propios de Internet, y que además ofrecen un espacio para configurar y organizar todo el material o recurso para el aprendizaje y lograr que el alumno adquiera conocimiento. A pesar de que existen diversas plataformas con las características antes mencionadas, una aplicación dotada de Web semántica es una de las mejores alternativas para implementar el aprendizaje en línea, ya que el uso de las ontologías tiene un gran potencial para este dominio debido a que una de sus principales características es que infieren conocimiento a partir de las definiciones dadas, es por eso que el desarrollo de la ontología presentada con base a los principios del E-learning facilita el proceso de evaluación de las asignaturas técnicocientíficas en el ITO.

\section{Trabajo a futuro}

Tomando en cuenta la importancia que tiene la educación como uno de los pilares fundamentales para el desarrollo de los países, como trabajo a futuro se pretende desarrollar una aplicación Web en la cual se implemente la ontología presentada en este 
Diseño de una ontología para el proceso de evaluación de las asignaturas técnico-científicas ...

trabajo. De igual manera, se pretende que dicha aplicación haga uso de los algoritmos de barajeo de preguntas y respuestas, con la particularidad de que, para la generación de respuestas, se definan y apliquen métodos para la generación de distractores, esto con base en los investigación que se ha realizado para este trabajo acerca de los errores más comunes que se comenten en evaluaciones de matemáticas. Lo que se buscará con esto es aportar facilidad a la elaboración de quiz (exámenes) correspondientes a materias del área de matemáticas (técnico-científicas), a partir de lo que la Web semántica, en específico las ontologías, puedan beneficiar a la educación.

\section{Referencias}

1. Antonio, L. R. D.: Entorno Web para la generación de reactivos en la evaluación de aplicación de derivadas. Tesis de Maestría, Departamento de posgrado e investigación, Instituto Tecnológico de Orizaba, Orizaba, Ver, Méx. (2014)

2. Baelo, A. B.: El E-learning, una respuesta educativa a las demandas de las sociedades del siglo xxi. España, Universidad de León (2009)

3. González, O. M.: Evaluación de opción múltiple v.s. evaluación tradicional, Un estudio de caso en ingeniería. Revista Virtual, Universidad Católica del Norte (2014)

4. Tabares, J. J., Jiménez, J. A.: Ontología para el proceso evaluativo en la educación superior. Revista Virtual, Universidad Católica del Norte, 42, pp. 68-79 (2014)

5. Ramos, E., Núñez, H.: Ontologías: componentes, metodologías, lenguajes, herramientas y aplicaciones. Caracas (2007)

6. Corcho, O., Fernández, M., Gómez, A., López, A.: Construcción de ontologías legales con la metodología METHONTOLOGY y la herramienta WebODE (2005)

7. Rodriguez, A., Hernandez, G., Colomo, R., Gomez, J. M., Garcia, A., Alor, G., Valencia, R.: Towards an Ontology to Support Semantics Enabled Diagnostic Decision Support Systems. Current Bioinformatics 7, No. 3, pp. 234-45 (2012)

8. Protégé: Protege.stanford.edu (2016)

9. Menéndez, V., Prieto, M., Zapata, A.: Sistemas de Gestión Integral de Objetos de Aprendizaje. Facultad de Matemáticas de la Universidad Autónoma de Yucatán (2010)

10. Clarenc, C. A.: Analizamos 19 plataformas de E-learning. Disponible en: www.congresoelearning.org, Grupo GEIPITE, Congreso Virtual Mundial de e - Learning (2013)

11. Tavangarian, D., Leypold, M. E., Nölting, K., Röser, M.: Is e-Learning the Solution for Individual Learning? University of Rostock, Germany (2004)

12. Bacon, D.: IMS Question and Test Interoperability. MSOR Connections, 3(3) (2003)

13. W3C.: w3.org. Disponible en: https://www.w3.org/TR/sparql11-query (2016) 